The parts I found most interesting were those on the problems inherent in the use of pesticides, antibiotics and the products of the green revolution.

The book ends with a list of suggestions for an ecologically virtuous life. Ironically one of the recommendations is to save paper by using both sides when writing letters: the authors don't mention how many trees had to be felled to produce their book.

Gillian MoORe

\section{Classify knowledge}

The Fabric of Knowledge: A Study of the Relations Between Ideas. By J. L. Jolley. Pp. 130. Duckworth: London, October 1973.) $£ 2.95$.

THIS monograph will be of interest to anyone concerned with the problems of organising scientific or technical documents. It is more specialised than the title suggests, being an examination of the use of integrative level theory for the organisation of concepts in scientific and technical subjects. There are three parts. The first recounts the development of Mr Jolley's ideas, the second supports them by means of set theory and the third is a brief summary of previous ideas applied in the organisation of knowledge.

The first section is the most satisfactory and least controversial. It should be read in conjunction with the account of similar work by the Classification Research Group in Classification for a General Index Language, by D. J. Foskett (Library Association, 1970). Despite the common starting point there seem to be considerable differences of detail in the two sets of ideas. The levels identified are not exactly the same and their method of use varies: the CRG uses them for organising entity concepts only, whereas $\mathrm{Mr}$ Jolley includes all concepts. His semantic types used within the levels also differ from the categories used by the CRG. Unfortunately a full comparison is not possible since $\mathrm{Mr}$ Jolley has not written at sufficient length to do himself justice. A lot more examples would have helped to clarify the sometimes eccentric terminology and definitions that in themselves are inadequate for complete understanding.

There is no reason to doubt the usefulness of Mr Jolley's analysis, since it is based on many years of work in indexing consultancy. Even the set theory may be useful, but the exposition here is difficult to understand. Whether it is really necessary is another matter: the CRG have managed without it. It could be that they have merely failed to make explicit the real theory underlying their work, but the experience with categories should be a warning against Mr Jolley's claim to objectivity. There are no generally accepted categories of reality, and their use in classification can only be judged pragmatically. The only way to be objective in the classification of ideas is to examine the nature of knowledge rather than the nature of some supposed objective reality.

There seems little ground for $\mathrm{Mr}$ Jolley's optimism about the extension of his theory beyond science and technology. Integrative level theory relates to natural objects and there is no obvious reason why it should provide a structure for philosophy, history, religion or the arts. It is sufficient that it has a use in science and Mr Jolley's work is welcome independent support for that of the CRG. Both his suggested pattern of ideas and the use of set theory are worthy of further research.

\section{W. LANGRIDGE}

\section{Analysis}

Elementary Real and Complex Analysis. By Georgi E. Shilov. Revised English edition translated and edited by Richard A. Silverman, Pp. xi+516. (Mathematical Analysis: Vol. 1). (MIT: Cambridge, Mass. and London, 1973.) $\$ 14.95$.

This translation, by R. A. Silverman is, as usual, of high standard. The appearance of the book is good, the printing and accuracy so meticulous, that an error on page 223, where $f\left(x_{0}\right)+\mathrm{h}$ should read $f\left(x_{0}+h\right)$, comes as a shock.

The book covers much of the analytical knowledge demanded of a mathematics graduate-real and complex numbers, vector functions, metric spaces, limits, convergence, elementary functions, the theorems of the calculus, Reimann (-Stieltjes) integrals, analytical function theory (for piecewise smooth curves) and improper integrals. The chapters on limits (using directions) and improper integrals are probably the most refreshing. Functions of several variables, measure and Lebesgue integration receive only passing reference.

The author's declared aim is an easier 'Dieudonné'; nevertheless mathematical sophistication, and hard thought about abstract ideas, are demanded of the reader particularly in the earlier chapters. This may prove a deterrent to weaker students, scientists and engineers for whom the book is also intended. There is a good collection of examples, some normally accorded theorem status, some difficult even with the hints of the appendix.

This is a scholarly book; carefully used it could provide a logical, integrated and economic presentation of basic work.

Margaret Jackson

\section{Domestic life in Africa}

Man and Woman among the Azande. Edited by E. E. Evans-Pritchard. Pp. 197. 8 plates. (Faber: London, February 1974.) $£ 4.50$.

Sir Edward Evans-Pritchard died towards the end of 1973, and this is the last book to come from his pen. As such it is appropriate that in it he returns to the study of the Azande people of Central Africa, the group about whom he first wrote more than forty years ago and about whose thought he published his first major work.

This book is a collection of Zande texts, some taken down in the 1920 s, some written more recently. EvansPritchard links these with brief comments of his own, and in a few places discusses and clarifies obscure passages. $\mathrm{He}$ is always careful to allow his Azande informants to speak for themselves and his editorial presence never prevents his reader from having to deal for himself with the complexities, contradictions and individualities of Zande thought and belief.

Evans-Pritchard was a great and respected social anthropologist, and it is probably due to his eminence that he was able to publish this book, for it is hard to believe that a commercial publisher would risk issuing a series of native observations about the problems and joys of domestic life if these were not supported by the name of such a famous author. But we must be thankful for that eminence, for it has made available a series of fascinating documents about the relations of men and women in a single exotic culture. Material such as this has rarely been published in book form before.

One of the main qualities of this book is that it shows the almost-raw material from which anthropologists huild up their analyses and abstractions. Women quarrel, men complain, an informant recalls a story heard about a neighbour, an old man criticises the conduct of the young. All this is faithfully and simply recorded here and it is left to the reader to make what he can of it. In some places he would clearly benefit from additional information or guidance from the editor, but this is not provided. To get the full worth of the book it is therefore necessary to read some of EvansPritchard's other writings on the Azande; once this is done the mass of data about male/female relations can be used more profitably.

In the final count anthropologists depend on the quality of their informants. Evans-Pritchard, like Malinowski and others before him, always stressed the need to record information verbatim; only in that way, he argued, could a true appreciation of native 